\title{
Physical Design Cognition: A Non-symbolic Formalization for Performing Design Knowledge
}

\author{
Daniel Smithwick \\ SUTD-MIT International Design Center, USA \\ djs2@mit.edu
}

\author{
Lawrence Sass \\ Massachusetts Institute of Technology, USA \\ Isass@mit.edu
}

\begin{abstract}
This paper frames design knowledge as formalizable physical action for developing embodied computational design skills that can more fully exploit current and future digital fabrication prototyping methods. Digitally integrated prototyping tools reveal the physicality of cognition in computational design activity; however, because current theories of design knowledge define cognition as a mental process, physical computation design skills remain underdeveloped. We identify symbolic formalization as the root of this problem. We present a non-symbolic action-based notation drawing from embodied cognition as an alternative model for design cognition. Designerly knowledge is discussed in terms of reflective action and epistemic action.
\end{abstract}

Keywords: Embodied design cognition; Physical computing; Human-computer interaction; Action notation; Motion tracking.

\section{Introduction}

In a world largely defined by digital processes that render knowledge to that which can be programmed on an iPad, we must question what design knowledge is, where it exists, and how to expand upon and improve it. Is it in our minds? Is it in our finger tips? Is it in our artifacts? Can it be embedded in our tools? Such questions lie at the intersection of design cognition and design computation, in other words-in how designers think about and do design in a formalized way.

This work builds on Smithwick and Sass (2012) in which they compare an electronic calculator with a logarithmic slide rule to investigate the experiential differences between using digital and analog computational tools, respectively. They speculate that digital computation limits the designer in the same way that digitally compressed music limits the listener. By extending the concept of visual computation which is "doing design directly through computations on shapes, rather than indirectly through computations on text or symbols" (Knight and Stiny, 2001, pg. 362), Smithwick and Sass suggest that tools that integrate physical computation may expand the designer's means for performing qualitative computations by framing design cognition in terms of the continuous nature of analogical calculating.

In order to develop physical computing design tools and production methods we need to expand our models of design knowledge beyond symbolic representation into embodied action. Early theories of design cognition were not only based on metaphors of the mind as a symbol processor, but were constructed as and tested with symbol processors (Newell and Simon, 1961). Design thinking became defined by the limitations of the very tools used to simulate design activity. This circular logic led to a design science (Simon, 1969) and research methods
(Eastman, 1970; Gero, 1994) resulting in computational design tools that neglected and limited development of the embodied intelligence involved in designing. This mentalist perspective of design cognition as mediated within computation is best summed up by Cross: “Asking 'Can a machine design?' is similar to asking 'Can a machine think?'” (2007, p. 58).

Alternative theories of human cognition such as Embodied Cognition and Situated Activity are not limited by the symbolic representation required when building information-theoretic models. Instead, "Knowledge depends on being in a world that is inseparable from our bodies, our language, and our social history" (Varela, Thompson, and Rosch, 1993, pg. 149). This work explores what design knowledge can be if we think through our bodies instead of through the mental 'aid' of our computational tools.

\section{The digital designer still uses his body}

Digital tools provide a good starting point for finding evidence of a designer's embodied intelligence because we can directly compare the symbolic description of a design activity with a non-symbolic description. For example, while the process of drawing a simple shape with a CAD tool can be symbolically described through text commands or algorithms, it can also be richly formalized through the physical motions of the body as it engages the tool (Figure 1).

These motions are embodied design intelligence in action. There is little reason to assume these continuous movements are any less formalizable than discrete algorithmic processes. A formalism that harnesses the feelings and characteristics of performing these movements-e.g., the speed, force, coordination, fluidity, among many potential others-is presented in this paper. 


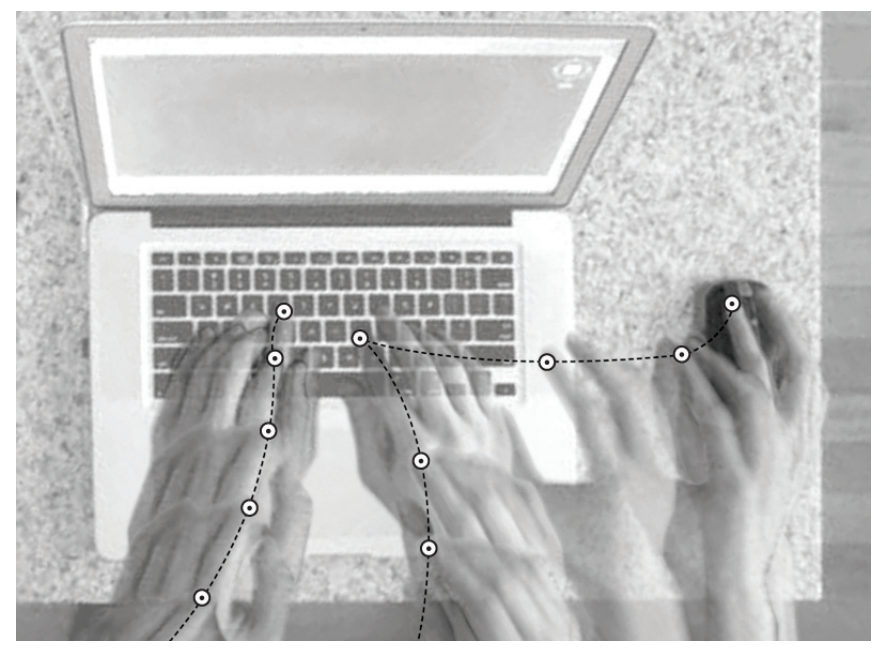

Figure 1: Motion tracking reveals the movements involved in drawing a circle with a CAD tool.

\section{Background}

\section{Design protocol analysis}

Design protocol studies have led to novel formalizations of the design process and have expanded models of design cognition (Dorst and Dijkuis, 1995). Protocol studies commonly represent the design process through coding schemas and segmentation (e.g., Kavakli and Gero, 2002); or as descriptive narratives made through video analysis (e.g., Suwa and Tversky, 1996); or from thoughts voiced by the designer known as the concurrent protocol method (e.g., Lloyd, Lawson, and Scott, 1995; Gero and McNeill, 1998). Many studies account for the physical actions designers make (e.g., sketching, looking, gesturing in Suwa, Purcell, and Gero, 1998); however, because the designer is framed as an information processing system her physical movements are considered a means to more fundamental design activity which occurs in the mind.

\section{Embodied Cognition}

Theories of Embodied Cognition (EC) can further expand our conception of design knowledge by inverting the information processing perspective-i.e., it is our physical actions that are the most fundamental activity. EC is highly theoretical drawing from the phenomenological philosophies of Husserl, Merleau-Ponty, and Heidegger (see Anderson, 2003; Wilson, 2002 for field review). Anderson states that the central focus of work in EC is the symbol-grounding problem which questions where symbols ultimately acquire meaning for humans. Anderson writes that symbols "must ultimately ground out in (terms of) the agent's embodied experience and physical characteristics" (2003, p. 2). In other words, while we can and do use symbols to represent and communicate design knowledge, these symbols ultimately depend on our bodies and the physical environment for us to make sense of them.

\section{Human-computer interaction}

Although most work in EC is theoretical there are a few notable exceptions demonstrating practical applications exploiting the physical characteristics of computational tools and abilities of the users (Dourish, 2001). Recent examples in Human-computer Interaction demonstrate theories applied in prototyping tools. Some tools situate the control and design interface of digital fabrication tools such as CNC routers directly on the machine and allow for real-time control of the cutting bit through motion tracking technologies (Willis et al, 2010; Willis et al, 2011). Zoran and Paradiso constructed a handheld Dremel-like digital sculpting tool called FreeD enabling a novice user to directly engage with the sculpting material through the aid of computational feedback to correct movement errors based on a digital design (2013).

These projects demonstrate how digitally integrated physical tools can engage the designer's body by means of haptic, motion, and infrared sensors which translate the bodily reactions into digital representations. Our intent here is to develop a framework for questioning what the meaningful bodily actions are for design in the first place and to propose a formalization for harnessing and improving such actions.

\section{A Non-Symbolic Notation for Music}

Research grounded in Performance Theory provides an example of how a non-symbolic formalization may work. Performance Theory shares with EC a foundation in phenomenology; however, primary focus is anthropological and not computational, prioritizing study of the body as a social, cultural, and physical phenomenon rather than a technical and mathematical system (Schechner, 2002).

Most applications of performance theory are found in the performing arts - dance, drama, action art, and music. We present a study from Music Performance Research (Orning, 2012) of German composer Helmut Lachenmann's 'action-based notation developed for his 1969 piece for solo cello, Pression (Figure 2). Orning describes Lachenmann's action notation as:

"A reversal of traditional hierarchies, prioritizing the performance over the musical text. Lachenmann shifts the focus from the score as musical text to the action embodied in performance. This shift in compositional focus calls for a complementary shift in analytical focus. And the focus on the act of performance lends itself to an analysis grounded in performance theory" (2012, p. 16). 

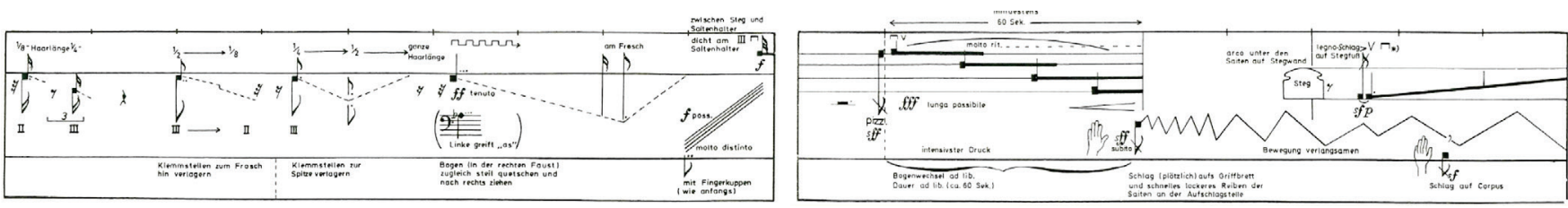

Figure 2: Action-based music notation developed by Lachenmann for 'Pression', 1969 (Orning, 2012).

In Lachenmann's notation, the musical notes as traditionally represented still exist and function accordingly; however, the notation makes evident a 'hidden dimension' between the notes that the musician can formally exploit. Lachenmann gives suggestions for how the musician might interpret and perform this musical dimension through different types of lines (zig-zags, dotted lines, sloping lines, horizontal arrows, etc.) and graphics (figurative drawings of hands and cello parts) (Figure 2).

With Lachenmann's action-based notation we have an example of a type of formalization that circumvents pre-determined symbolic representation without sacrificing the structure of a framework that makes it possible to share, preserve, and improve an active form of knowledge.

\section{A Non-symbolic Notation for Design}

This paper shifts the traditional hierarchies found in design cognition and computation which place priority on symbolic schemas and processes as the foundation of creative work in much the same way that traditional hierarchies in music place priority on the musical text. To illustrate our action-based formalization we return to the example of drawing a shape with a CAD tool. To more clearly convey our formalization we contrast it with a symbolic formalization of the same exercise of drawing a circle in AutoCAD (Table 1).

The formalization is to be read from left to right as one might read musical notation. In Step 1, the horizontal dashed line represents the line made by the fingers as they rest on the 'home row' of a standard computer keyboard. The up and down arrows indicate the order, direction, and quality in which the designer can move his fingers as they strike the keys to spell out the word 'circle'. For example, in Step 1 the left-most black down arrow indicates to the designer that using his left index finger (labeled 1L) he should strike the ' $C$ ' key on the keyboard. Next, the designer should use his middle finger on his right hand (labeled $2 \mathrm{R}$ ) to strike the ' $\mathrm{l}$ ' key on the computer keyboard. Further steps of the action notation for using the mouse to complete the drawing exercise are omitted.

\section{Designerly ways of unknowingly using the body}

In contrast to traditional design protocol analyses, this formalization of design activity can be used to improve designers' physical abilities. Physical computation can harness the performative qualities of design knowledge as expressed through the physical actions of the designer's movements. For example, by formalizing characteristics of physical movement-the timing between movements, the amount of force used, or the duration of striking a key designers would be more able to directly associate, manipulate, and disseminate the physical feelings associated with particular design moves (Table 1). This is a potential 'hidden dimension' of digital design.

If so, it opens up an avenue of investigation overlooked in design cognition studies: how designers use their bodies as aids to explore novel solutions in computational design activity. Kirsh and Maglio developed the notion of Epistemic Action in cognitive science to study this phenomenon. They witnessed expert Tetris players utilize "physical actions [to] make mental computation easier, faster, or more reliable" (1995, p. 513). The players would perform seemingly extraneous physical actions during game play, such as overly rotating zoids or translating zoids across the entire screen, taking them further away from achieving the immediate goal at hand-dropping the zoids into rows. But ultimately such actions "make it easier for [Tetris players] to attend, recognize, generate and test candidates, and improve execution" (p. 548).

Epistemic actions can be contrasted to Schon's reflective actions in light of Cross's mentalist notion of design knowledge. Schon describes a phenomenon in design activity he calls 'move experiments' as a part of a designer's reflective actions. He writes, that designers "attend to processes that computers are unable- at least presently unable - to reproduce: the perception of figures or gestalts, the appreciation of qualities, the recognition of unintended consequences of moves" (Schon and Wiggins, 1992, p. 156). Schon's reflective actions are mental activities for which he challenges researchers to develop computational tools to support. On the other hand, epistemic actions are physical activities that precede and bring about such mental activity. We speculate that future computational tools will support and enhance the performative epistemic actions for facilitating creative thought in addition to the analytic reflective actions.

\section{Future Work}

Prototyping methods that integrate digital fabrication devices will benefit from physical design cognition (e.g., the methods of Sass and Smithwick, 2013). Bodily actions facilitate learning and design improvement during the assembly process of iterative digital design studies (Figure 4) in much the same way that physically practicing a musical instrument aids the musician in performing a musical score. 
Table 1: Comparison between a symbolic formalization with an action-based notation for drawing a circle in AutoCAD.

$\begin{aligned} & \text { Symbolic Formalization } \\ & \text { (AutoCAD) }\end{aligned}$
Initialize the Command
$\begin{aligned} & \text { 1. Using the finger notation, type } \\ & \text { the keys 'c-i-r-c-l-e' on the } \\ & \text { keyboard. } \\ & \text { 2. Hit the 'spacebar' key on } \\ & \text { keyboard. }\end{aligned}$

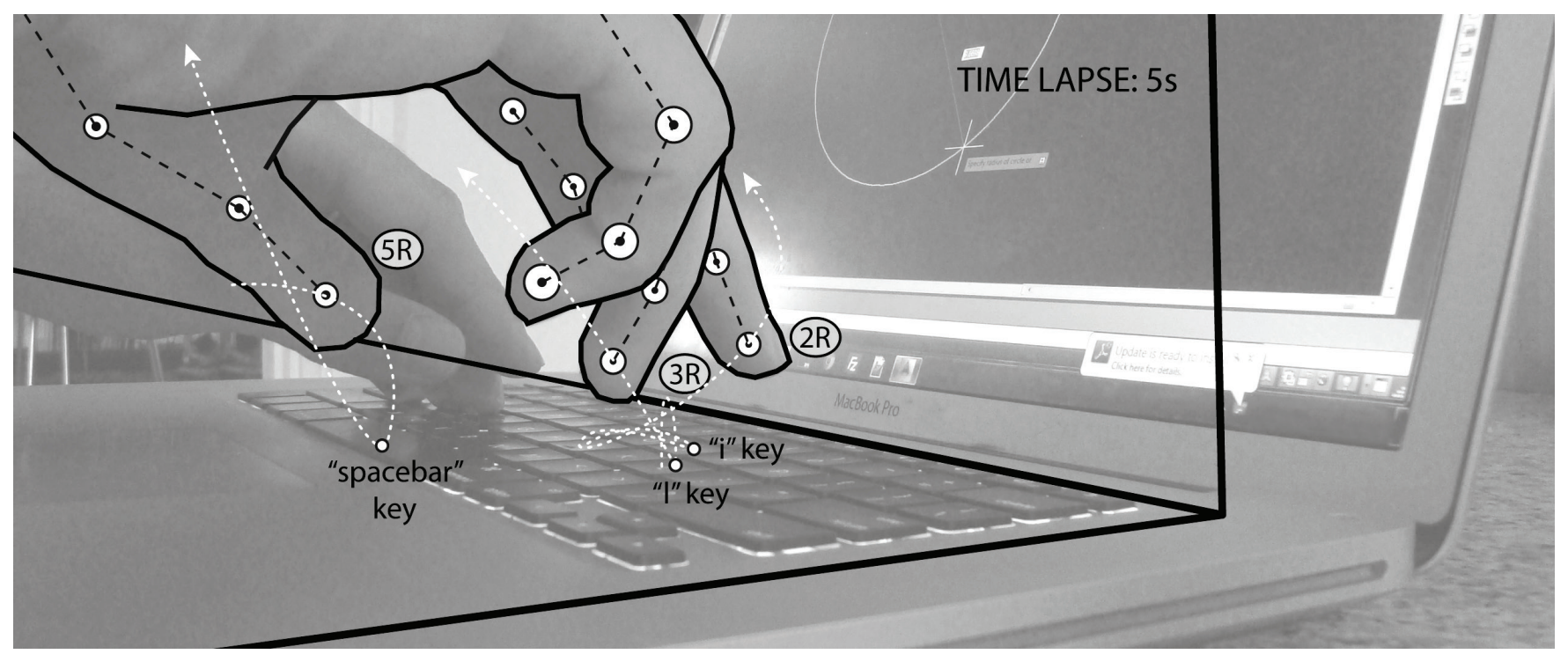

Figure 3: Detail of articulated right hand movements while typing "circle" in AutoCAD.

Formalizations of these bodily actions will expand the means of learning processes and could provide a framework for developing novel robotic digital fabrication aids (Wallis, et al, 2010).

In addition, improvement can be made to the methods in which we study a designer's physical actions. Future projects should utilize high resolution motion capture technologies to reveal more subtle actions in the design process that can be exploited and formalized (as speculated in Figure 3).

Lastly, research is needed to explore the differences between the embodied intelligence of novice and expert designers. A better understanding of these differences can lead to physical training techniques suitable for design technology curriculum at all levels.

\section{Conclusion}

The designer's body is a powerful tool for thinking and expressing ideas that has gone neglected in design computation research. Design cognition no longer needs to be limited to what can be programmed on an iPad; it can be as physically impressive as an Olympic gymnast; as emotionally moving as an improvisational jazz pianist; and as graceful as a dancer. 


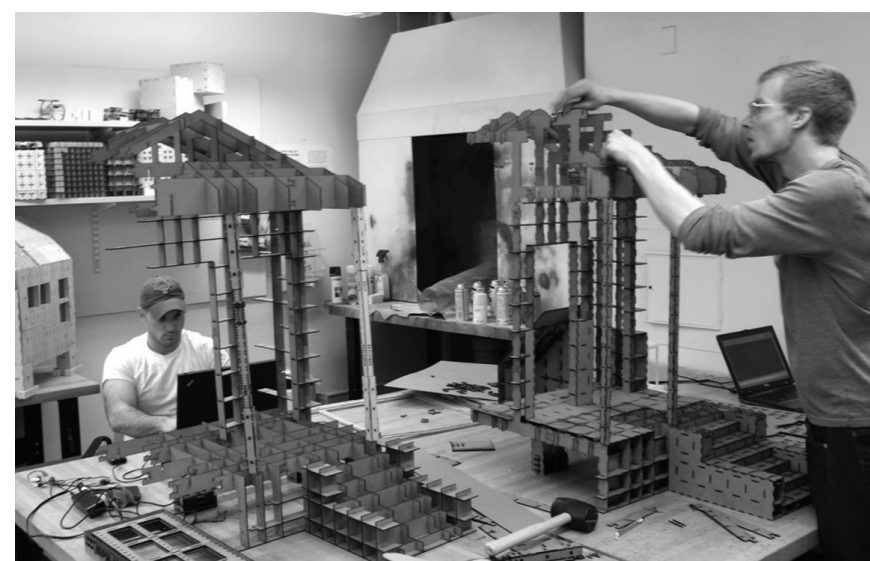

Figure 4: Assembly of study models for MoMA's Digitally Fabricated House for New Orleans project, 2008.

\section{Acknowledgements}

Funding for this research was provided by the SUTD-MIT International Design Center. Much of this work was conceived during the lead author's experience of playing drums with the MIT Festival Jazz Ensemble and attending lectures in George Stiny's Research Seminar in Design Computation.

\section{References}

Anderson, M. (2003). Embodied Cognition: A field guide, Artificial Intelligence, 149, 1, pp. 91.

Cross, N. (2007). Natural Intelligence in Design, in Nigel Cross (ed.) Designerly Ways of Knowing, (pp. 49-62) Basel: Birkhauser.

Dorst, K., Dijkhuis, J. (1995). Comparing paradigms for describing design activity, Design Studies, Volume 16, Issue 2, Pages 261-274.

Dourish, P. (2001). Seeking a Foundation for Context-Aware Computing. Human Computer Interaction, Vol 16, pg. 229-241.

Eastman, C. (1970). On the Analysis of Intuitive Design Processes, in Moore, G. (ed.) Emerging Methods in Environmental Design and Planning, pp. 21-37. MIT Press: Cambridge.

Gero, J. (1994). Computational Models of Creative Design Processess, in Dartnall (ed.) Artificial Intelligence and Creativity, Klower, Dordrecht, the Netherlands.

Kavakli, M and Gero, J. (2002). The Structure of Concurrent Cognitive Actions: a case study on novice and expert designers'. Design Studies Vol 23, pg. 25-40.

Kirsh, D., and Maglio, P. (1995). On Distinguishing Epistemic from Pragmatic Action. Cognitive Science, 18, pp. 513-549.
Knight, T. and Stiny, G. (2001) Classical and Non-classical Computation. Information Technology, Vol 5 No. 4, pp 355-372.

Newell, A. and Simon, H. (1961) "Computer Simulation of Human Thinking," Science 134: 2011-17.

Orning, Tanja. (2012) "Pression - a performance study." Music Performance Research. Vol 5, 12-31.

Sass, L., Smithwick, D. (2013). Mass Customised Structures for Relief: Physical production with Digital Fabrication. In Piroozfar and Piller (eds.), Mass Customisation and Personalisation in Architecture and Construction. (pp. 174-183). London: Routledge.

Schechner, R. (2002). Performance Studies: an Introduction, $1^{\text {st }}$ Edition. Routledge, New York.

Schon, D., Wiggins, G. (1992). Kinds of Seeing and their functions in designing. Design Studies Vol 13 No 2.

Simon, H. (1969). The Sciences of the Artificial, 1st Edition. MIT Press, Cambridge.

Smithwick, D., Sass, L. (2012). Designing in HiFi: Digital Fabrication for Physical Computation. Proceedings from Sigradi, November 13-16,pg 622-624.

Suwa, M. and Tversky, B. (1996). What architects see in their design sketches: implications for design tools. Human factors in computing systems: CHI'96, ACM. New York, pp. 191-192.

Suwa, M., Purcell, T., and Gero, J. (1998). Macroscopic analysis of design processes based on a scheme for coding designers' cognitive actions. Design Studies, Vol 19, pp. 455-483.

Varela, F.,Thompson, E.,Rosch, R (1993). The Embodied Mind: Cognitive Science and the Human Experience. Cambridge, MA: MIT Press.

Wallis, M., Popat, S., Mckinney, J., Bryden, J., and Hogg, D. (2010). Embodied Conversations: Performance and the design of a robotic dancing partner. Design Studies, Vol 31, pg. 99-117.

Willis, K., Lin, J., Mitani, J, and Igarashi, T. (2010). Spatial Sketch: Bridging Between Movement \& Fabrication. TEI '10 ACM, January 24-27, Cambridge, USA.

Willis, K., Xu, C., Wu, K., Levin, G., and Gross, M. (2011). Interactive Fabrication: New Interfaces for Digital Fabrication. Tangible, Embedded, and Embodied Interaction Conference, January 22-26, Portugal.

Wilson, M. (2002). Six Views of Embodied Cognition. Psychonomic Bulletin and Review, vol 9 (4), pg 625-636.

Zoran, Amit and Joseph A. Paradiso. (2013) "FreeD - A Freehand Digital Sculpting Tool." The 31th international conference extended abstracts on Human factors in computing systems (CHI '13). ACM, Paris, France. 